\title{
Gamma-ray emission from massive young stellar objects
}

\author{
A. T. Araudo ${ }^{1,2, \star}$, G. E. Romero ${ }^{1,2, \star \star}$, V. Bosch-Ramon ${ }^{3}$, and J. M. Paredes ${ }^{4}$ \\ 1 Instituto Argentino de Radioastronomía, C.C.5, (1894) Villa Elisa, Buenos Aires, Argentina \\ e-mail: aaraudo@fcaglp.unlp.edu.ar \\ 2 Facultad de Ciencias Astronómicas y Geofísicas, Universidad Nacional de La Plata, Paseo del Bosque, 1900 La Plata, Argentina \\ 3 Max Planck Institut für Kernphysik, Saupfercheckweg 1, Heidelberg 69117, Germany \\ ${ }^{4}$ Departament d'Astronomia i Meteorologia, Universitat de Barcelona, Martí i Franquès 1, 08028, Barcelona, Spain
}

Received 12 April 2007 / Accepted 18 August 2007

\section{ABSTRACT}

\begin{abstract}
Context. Massive stars form in dense and massive molecular cores. The exact formation mechanism is unclear, but it is possible that some massive stars are formed by processes similar to those that produce the low-mass stars, with accretion/ejection phenomena occurring at some point of the evolution of the protostar. This picture seems to be supported by the detection of a collimated stellar wind emanating from the massive protostar IRAS 16547-4247. A triple radio source is associated with the protostar: a compact core and two radio lobes. The emission of the southern lobe is clearly non-thermal. Such emission is interpreted as synchrotron radiation produced by relativistic electrons locally accelerated at the termination point of a thermal jet. Since the ambient medium is determined by the properties of the molecular cloud in which the whole system is embedded, we can expect high densities of particles and infrared photons. Because of the confirmed presence of relativistic electrons, inverse Compton and relativistic Bremsstrahlung interactions are unavoidable.

Aims. We aim to make quantitative predictions of the spectral energy distribution of the non-thermal spots generated by massive young stellar objects, with emphasis on the particular case of IRAS 16547-4247.

Methods. We study the high-energy emission generated by the relativistic electrons which produce the non-thermal radio source in IRAS 16547-4247. We also study the result of proton acceleration at the terminal shock of the thermal jet and make estimates of the secondary gamma rays and electron-positron pairs produced by pion decay.

Results. We present spectral energy distributions for the southern lobe of IRAS 16547-4247, for a variety of conditions. We show that high-energy emission might be detectable from this object in the gamma-ray domain. The source may also be detectable in X-rays through long exposures with current X-ray instruments.

Conclusions. Gamma-ray telescopes such as GLAST, and even ground-based Cherenkov arrays of new generation can be used to study non-thermal processes occurring during the formation of massive stars.
\end{abstract}

Key words. stars: formation - gamma rays: theory - stars: individual: IRAS 16547-4247

\section{Introduction}

The mechanism of formation of massive stars remains one of the open questions in the field of star formation. It is known that these stars originate inside massive molecular clouds but the sequence of processes which take place during the formation of the star are mostly unknown. It has been suggested, for example, that the coalescence of various protostars in the same cloud can lead to the emergence of a massive star (e.g. Bonnell et al. 1998). Massive stars appear in massive stellar associations where cloud fragmentation seems to be common. Alternatively, a massive star could form by the collapse of the core of a massive cloud, with associated episodes of mass accretion and ejection, as observed in low-mass stars (e.g. Shu et al. 1987). In such a case, the effects of jets propagating through the medium that surrounds the protostar should be detectable.

Recently, Garay et al. (2003) detected a triple radio continuum source associated with the protostar IRAS 16547-4247. The radio source presents a linear structure consisting of a thermal core, and two radio lobes. The southern lobe is clearly non-thermal, indicating the presence of relativistic electrons

\footnotetext{
$\star$ Fellow of CONICET, Argentina.

$\star \star$ Member of CONICET, Argentina.
}

that produce the observed radiation by synchrotron mechanism. This non-thermal source has been interpreted by Garay et al. (2003) as the termination point of one of the jets ejected by the protostar. There, a strong shock would accelerate the electrons to relativistic energies by the Fermi mechanism (e.g. Bell 1978). The observed spectral index of $\alpha \sim-0.6\left(S_{v} \propto v^{\alpha}\right)$ is in good agreement with what is expected from an uncooled population of relativistic electrons produced by diffusive shock acceleration at a strong non-relativistic shock (e.g. Protheroe 1999).

The angular separation of the non-thermal source from the core corresponds to a linear distance of only $0.14 \mathrm{pc}$, and then the population of relativistic particles is inside the molecular cloud. Hence, these particles are in a rich environment, with a high density of ambient matter and photon field from the infrared emission of the cloud. Inverse Compton (IC) and relativistic Bremsstrahlung losses are then unavoidable for these particles. If protons are accelerated at the termination shock along with the electrons, then inelastic $p p$ collisions can take place, producing pions, which will decay yielding gamma rays, relativistic electron-positron pairs and neutrinos. The radiation produced via all these mechanisms is likely to be steady at scales of years due to the dynamical timescales of the processes occurring at the source. 
The main goal of this paper is to estimate the high-energy yield of all these interactions, both leptonic and hadronic, in order to ponder whether gamma-ray astronomy can be used to probe massive star formation and the outflows it could produce. Untill now, thermal radio and X-ray emission has been associated with the formation of low-mass stars. Here we show that massive protostars can produce a significant amount of radiation in the gamma-ray domain, because of the dense and rich medium in which they are formed.

The structure of the paper is as follows. In the next section we provide the basic information about IRAS 16547-4247, the associated radio sources, and the ambient medium. Then, in Sect. 3 we discuss the particle acceleration and the different losses for the relativistic particles in the southern lobe of the radio source. Section 4 deals with the gamma-ray production. Our results are presented in the form of spectral energy distributions (SEDs), for different sets of parameters. We close with a brief discussion and a summary in Sect. 5.

\section{The protostar IRAS $16547-4247$ and its host environment}

The source IRAS 16547-4247 corresponds to a young massive star-forming region, associated with an O-type protostar, located at $2.9 \mathrm{kpc}$ (Garay et al. 2003). The luminosity of the source is $L \sim 6.2 \times 10^{4} L_{\odot} \approx 2.4 \times 10^{38} \mathrm{erg} \mathrm{s}^{-1}$, peaking at the infrared, which makes it the most luminous detected young stellar object (YSO) with thermal jets. Brooks et al. (2005) reported the detection of a chain of $\mathrm{H}_{2} 2.12 \mu \mathrm{m}$ emission knots which trace the collimated outflow that emanates from the center of the source. The SED of the IR emission can be described by a modified blackbody function with a peak temperature of $30 \mathrm{~K}$. The total mass of the cloud is $M_{\mathrm{cl}}=9 \times 10^{2} M_{\odot}$ (Garay et al. 2003). Molecular line observations indicate that the size of the cloud is $\sim 0.38 \mathrm{pc}$ in diameter $\left(\approx 1.1 \times 10^{18} \mathrm{~cm}\right)$. If we assume a spherical geometry, the averaged particle (atoms of $\mathrm{H}$ ) density of the cloud is $n_{\mathrm{cl}} \approx 5.2 \times 10^{5} \mathrm{~cm}^{-3}$. The energy density of IR photons in the cloud, assuming homogeneous distribution, is $w_{\mathrm{ph}} \approx 1.8 \times 10^{-9} \mathrm{erg} \mathrm{cm}^{-3}$.

The radio observations made by Garay et al. (2003) with the ATCA and the deeper observations by Rodríguez et al. (2005) with the VLA show the existence of a triple radio source inside the molecular cloud. The three components of the radio source are aligned in the northwest-southeast direction, with the outer lobes separated from the core by a projected distance of $0.14 \mathrm{pc}$. The central source is elongated and has a spectral index of $0.33 \pm 0.05$, consistent with free-free emission from a collimated jet (Rodríguez et al. 2005). The radio lobes have some substructure. The integrated emission from the northern lobe has a spectral index of $-0.32 \pm 0.29$, of dubius thermal/non-thermal nature. On the other hand, the spectrum of the southern lobe radiation has an index $\alpha=-0.59 \pm 0.15$. In fact, the shock heated material can radiate sufficiently so as to ionize the surrounding medium, and the free-free absorption could modify to some extent the radio spectrum. However, we do not have enough data to characterize the region and to derive either its thermal properties or the ionization degree. Otherwise, diffusive shock acceleration by strong non-relativistic shocks naturally produces a power-law particle distribution with an index similar to what is inferred from the observations. Thus, we take as a first order approximation the observed radio spectrum as the original one, which would correspond to a non-thermal particle distribution. The inferred linear size for this lobe is $\approx 1.1 \times 10^{16} \mathrm{~cm}$. Nonthermal radio emission has been associated in the past with the

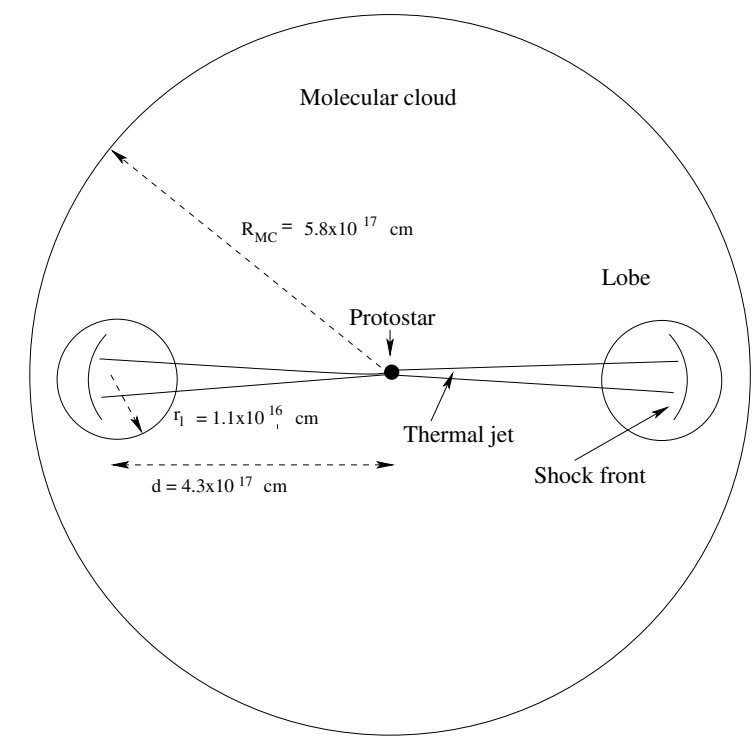

Fig. 1. Sketch of the scenario discussed in this work.

outflows of a few massive YSOs (see Rodríguez et al. 2005, and references therein). In no case the molecular cloud is as massive and luminous as for IRAS 16547-4247. The flux density of the southern lobe is $2.8 \pm 0.1 \mathrm{mJy}$ at $8.46 \mathrm{GHz}$. A sketch of the scenario discussed in this paper is shown in Fig. 1.

\section{Particle acceleration and losses}

At the termination point of the thermal jet a strong shock front is expected to be formed. Charged particles can be accelerated at the shock, with an acceleration rate $\propto \eta B$ (see below), where $B$ is the magnetic field and the efficiency $\eta$ depends on the acceleration mechanism and its details. In the case of diffusive shock, acceleration $\eta \sim\left(v_{\mathrm{s}} / c\right)^{2} / f_{\mathrm{sc}}$, where $v_{\mathrm{s}}$ is the shock velocity and $f_{\mathrm{sc}}$ is the ratio of the mean free path of particles to their gyro-radius. Close to the Bohm limit $f_{\mathrm{sc}} \sim 1$. The terminal velocity of the collimated outflow in IRAS 16547-4247 is unknown, however Martí et al. (1995) determined a velocity in the range $600-1400 \mathrm{~km} \mathrm{~s}^{-1}$ for the $\mathrm{HH} 80-81$ thermal radio jet, which is also powered by a massive YSO. Adopting a value of $\sim 1000 \mathrm{~km} \mathrm{~s}^{-1}$ for IRAS $16547-4247$, we have a reasonable efficiency of $\eta \sim 10^{-5}$ for the southern lobe of this source. The efficiency in the northern lobe would be lower, since the nonthermal emission does not seem to be dominant there.

The average matter density of the cloud is $5.2 \times 10^{5} \mathrm{~cm}^{-3}$. It is noted nevertheless that in the shocked material the density should be $\sim 4$ times this value. In our calculations of the Bremsstrahlung and pion-decay radiation we have adopted this higher density.

As mentioned above, the rate of energy gain for electrons at the acceleration region is:

$\dot{\gamma}_{\mathrm{e}, \text { gain }}=\frac{\eta e B c}{m_{\mathrm{e}} c^{2}}$,

where $\gamma$ is the Lorentz factor of the particle, and $m_{\mathrm{e}}$ is the electron rest mass. Similarly, for protons,

$\dot{\gamma}_{\mathrm{p}, \text { gain }}=\frac{\eta e B c}{m_{\mathrm{p}} c^{2}}$. 
Table 1. Magnetic field and normalization constants for the different cases considered in the text.

\begin{tabular}{ccccc}
\hline \hline$a$ & $\begin{array}{c}B \\
{[\mathrm{G}]}\end{array}$ & $\begin{array}{c}K_{\mathrm{e}_{1}} \\
{\left[\mathrm{erg}^{\Gamma_{\mathrm{e}_{1}}-1} \mathrm{~cm}^{-3}\right]}\end{array}$ & $\begin{array}{c}K_{\mathrm{p}} \\
{\left[\mathrm{erg}^{\Gamma_{\mathrm{p}}-1} \mathrm{~cm}^{-3}\right]}\end{array}$ & $\begin{array}{c}K_{\mathrm{e}_{2}} \\
{\left[\mathrm{erg}^{\mathrm{e}_{2}-1} \mathrm{~cm}^{-3}\right]}\end{array}$ \\
\hline 0 & $2.0 \times 10^{-3}$ & $2.6 \times 10^{-9}$ & - & - \\
1 & $2.5 \times 10^{-3}$ & $2.0 \times 10^{-9}$ & $6.7 \times 10^{-9}$ & $6.5 \times 10^{-10}$ \\
100 & $3.0 \times 10^{-3}$ & $2.7 \times 10^{-11}$ & $1.7 \times 10^{-8}$ & $1.5 \times 10^{-9}$ \\
\hline
\end{tabular}

\subsection{Non-thermal radiation losses and maximum energies}

In order to obtain the maximum energy of the primary particles we have to balance the energy gain and loss rates. In the case of electrons, the relevant losses are synchrotron, IC, and relativistic Bremsstrahlung losses. The latter two, under the conditions described in the previous section, are given by:

$\dot{\gamma}_{\mathrm{IC}}=-3.2 \times 10^{-17} \gamma^{2} \mathrm{~s}^{-1}$,

$\dot{\gamma}_{\mathrm{Br}}=-7.4 \times 10^{-10} \gamma \mathrm{s}^{-1}$.

The expression for the synchrotron losses depends on the magnetic field $B$ and is given below. We can estimate the magnetic field assuming equipartition in energy density between field and particles in the radio lobe, so that:

$\frac{B^{2}}{8 \pi}=u_{\mathrm{e}_{1}}+u_{\mathrm{p}}+u_{\mathrm{e}_{2}}$.

Here, $u_{\mathrm{e}_{1}}$ is the energy density of primary electrons, $u_{\mathrm{p}}$ that of primary protons, and $u_{\mathrm{e}_{2}}$ corresponds to secondary pairs that will result from the charged pions produced in inelastic $p p$ collisions. In each case $\left(i=\mathrm{e}_{1}, p, \mathrm{e}_{2}\right)$ :

$u_{i}=\int E_{i} n\left(E_{i}\right) \mathrm{d} E_{i}$,

where $n\left(E_{i}\right)=K_{i} E_{i}^{-\Gamma_{i}} \exp \left(-E_{i} / E_{i}^{\max }\right)$ is the particle density distribution $\left[\mathrm{cm}^{-3} \mathrm{erg}^{-1}\right]$.

It is a fact that there are relativistic electrons or pairs in the southern lobe. This population of relativistic leptons can be produced via acceleration either of electrons or protons. In the former case, the accelerated electrons would be responsible for the production of the detected synchrotron emission. In the latter case, accelerated protons would suffer $p p$ interactions with nuclei of the ambient medium generating charged pions which would decay to muons, which would subsequently decay to pairs. These pairs would then be the emitters of the observed non-thermal radiation. Although the calculation of the neutrino emission is out of the scope of this work, we note that neutrino luminosities would be similar to those of $\pi^{0}$-decay gamma-rays.

The most likely scenario is one with acceleration of both electrons and protons. Nevertheless, the relative number of accelerated protons is unknown, so we will write $u_{\mathrm{p}}=a u_{\mathrm{e}_{1}}$ and then $u_{\mathrm{e}_{2}}=f u_{\mathrm{p}}$. The value of $f$ was estimated using the average ratio of the number of secondary pairs to $\pi^{0}$-decay photons (Kelner et al. 2006). We will consider three cases: $a=0$ (no proton acceleration), $a=1$ (equal energy density in protons as in electrons), and $a=100$ (proton dominance, as is the case in the Galactic cosmic rays).

For a distribution of protons $n\left(E_{\mathrm{p}}\right)$, the energy spectra of secondary electron-positron pairs was calculated using the new parametrization of the inelastic cross-section of $p p$ interactions given by Kelner et al. (2006):

$\sigma_{\text {inel }}\left(E_{\mathrm{p}}\right)=34.3+1.88 L+0.25 L^{2} \mathrm{mb}$,
Table 2. Break energy in the electron spectra for different cases discussed in the text. Normalization constants for the power-law distributions above the break energy are shown.

\begin{tabular}{cccc}
\hline \hline$a$ & $\begin{array}{c}E_{\mathrm{b}} \\
{[\mathrm{eV}]}\end{array}$ & $\begin{array}{c}K_{\mathrm{e}_{1}}^{\prime} \\
{\left[\mathrm{erg}^{\Gamma_{\mathrm{e}_{1}}} \mathrm{~cm}^{-3}\right]}\end{array}$ & $\begin{array}{c}K_{\mathrm{e}_{2}}^{\prime} \\
{\left[\mathrm{erg}^{\Gamma_{\mathrm{e}_{2}}} \mathrm{~cm}^{-3}\right]}\end{array}$ \\
\hline 0 & $9.4 \times 10^{9}$ & $3.7 \times 10^{-11}$ & - \\
1 & $3.1 \times 10^{10}$ & $9.8 \times 10^{-11}$ & $3.2 \times 10^{-11}$ \\
100 & $2.2 \times 10^{10}$ & $9.5 \times 10^{-13}$ & $5.4 \times 10^{-11}$ \\
\hline
\end{tabular}

where $L=\ln \left(E_{\mathrm{p}} / 1 \mathrm{erg}\right)$. In the cases $a=0$ and $a=1$, $\Gamma_{\mathrm{e}_{1}}=2.18$, from the radio observations. For $a=1$, when primary electrons are still dominant, the same slope is assumed for them and for protons. Then, for the secondaries, we get $\Gamma_{\mathrm{e}_{2}}=2.13$. In the case of $a=100$, where the secondary leptonic emission dominates, $\Gamma_{\mathrm{e}_{2}}$ must be 2.18 and then $\Gamma_{\mathrm{p}}=\Gamma_{\mathrm{e}_{1}}=2.27$ for the primary particles.

Using the standard synchrotron formulae (Ginzburg \& Syrovatskii 1964) and the data from Rodríguez et al. (2005), along with the equipartition condition and the equations given above, we get the magnetic fields and the normalization constants shown in Table 1 , for each case considered. Typically, $B$ is about few $10^{-3} \mathrm{G}$.

Now, with this magnetic field we can calculate the synchrotron losses in each case:

$\dot{\gamma}_{\text {synchr }}(a=0)=-1.0 \times 10^{-14} \gamma^{2} \mathrm{~s}^{-1}$,

$\dot{\gamma}_{\text {synchr }}(a=1)=-1.1 \times 10^{-14} \gamma^{2} \mathrm{~s}^{-1}$,

$\dot{\gamma}_{\text {synchr }}(a=100)=-3.3 \times 10^{-14} \gamma^{2} \mathrm{~s}^{-1}$.

The different losses for electrons are shown in Fig. 2, along with the acceleration rate, for a value of $B$ of $2.5 \times 10^{-3} \mathrm{G}$ (case $a=1$ ). We can see that low energy electrons cool mainly through relativistic Bremsstrahlung, whereas the losses of the most energetic ones are dominated by synchrotron radiation. The dominant cooling regime changes at $E_{\mathrm{e}}=E_{\mathrm{b}}$, where $E_{\mathrm{e}}$ is the electron energy, either of primaries or secondaries. For a steady state, under the conditions considered here, the electrons will have an index $\Gamma_{\mathrm{e}_{1}, \mathrm{e}_{2}}$ for energies $E_{\mathrm{e}}<E_{\mathrm{b}}$ and $\Gamma_{\mathrm{e}_{1}, \mathrm{e}_{2}}+1$ for $E_{\mathrm{e}}>E_{\mathrm{b}}$. In Table 2 we show the values of $E_{\mathrm{b}}$ for the different cases under consideration, along with the corresponding normalization constants at high energies.

The relevant losses for protons are from inelastic $p p$ collisions with the cloud material. In our case, these losses are approximately given by (Mannheim \& Schlickeiser 1994):

$\dot{\gamma}_{p p}=-9.2 \times 10^{-10}\left[0.95+0.06 \ln \left(\frac{\gamma}{1.1}\right)\right] \gamma \mathrm{s}^{-1}$,

where now $\gamma$ is the Lorentz factor of the protons. Equating with Eq. (2), we obtain the maximum energy of the accelerated protons, with results of the order of $\sim 10^{15} \mathrm{eV}$. Although the gyroradius of these particles can be contained in the acceleration 


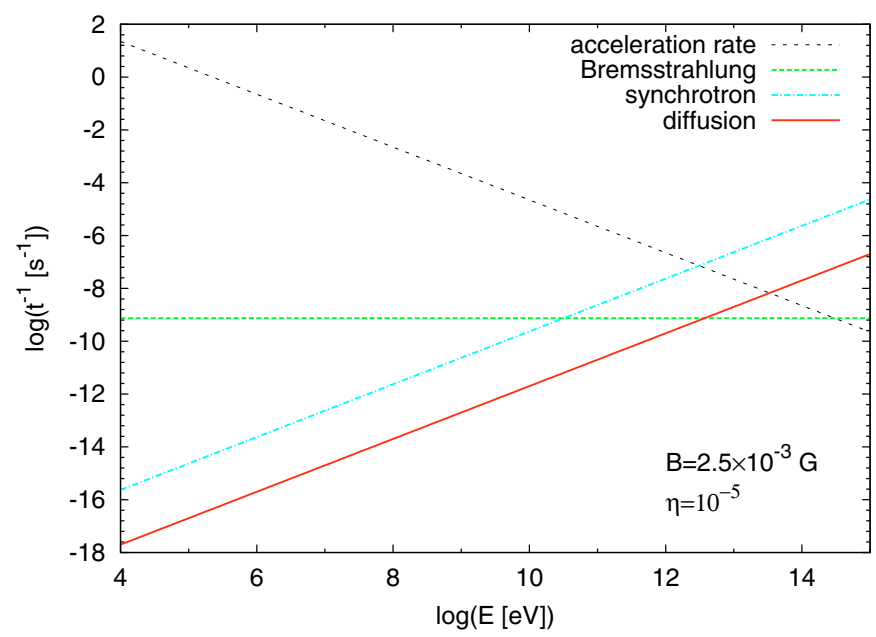

Fig. 2. Energy loss rates for synchrotron and relativistic Bremsstrahlung processes. The IC energy loss rate is not shown since it is negligible. The acceleration rate and the particle rate of diffusion out of the emitting region are also shown.

Table 3. Maximum energies obtained for electrons and protons accelerated in the southern lobe of the radio source associated with IRAS 16547-4247. The maximum energy for secondary pairs is shown in the last column.

\begin{tabular}{cccc}
\hline \hline$a$ & $\begin{array}{c}E_{\mathrm{e}_{1}}^{\max } \\
{[\mathrm{eV}]}\end{array}$ & $\begin{array}{c}E_{\mathrm{p}}^{\max } \\
{[\mathrm{eV}]}\end{array}$ & $\begin{array}{c}E_{\mathrm{e}_{2}}^{\max } \\
{[\mathrm{eV}]}\end{array}$ \\
\hline 0 & $3.1 \times 10^{12}$ & - & - \\
1 & $3.1 \times 10^{12}$ & $4.7 \times 10^{13}$ & $1.1 \times 10^{12}$ \\
100 & $2.8 \times 10^{12}$ & $5.7 \times 10^{13}$ & $1.8 \times 10^{12}$ \\
\hline
\end{tabular}

region, they can diffuse out of the cloud before reaching the mentioned maximum energy, hence a more tight constraint on the maximum energy can be obtained from the following equation:

$\frac{E_{\mathrm{p}}}{\eta e B c}=\frac{r_{\text {lobe }}^{2}}{D}$,

where $D$ is the diffusion coefficient and $r_{\text {lobe }} \approx 1.1 \times 10^{16} \mathrm{~cm}$ the size of the acceleration region. This assumption is supported by the fact that particles lose all their energy before they diffuse out of the emitting region (i.e. $\tau_{\text {cooling }}<\tau_{\text {diff }}$ ) (see Fig. 2). Working on the Bohm limit we can set $D=D_{\mathrm{B}}=r_{\mathrm{g}} c / 3$, with $r_{\mathrm{g}}$ the gyroradius. In such a case we get the maximum energies shown in Table 3, which are more than one order of magnitude lower than in the previous calculation.

\subsection{Relativistic particle distribution}

The lepton particle distribution in the emitting region can be calculated, as a first order approximation, adopting a one-zone model, in which particles, once injected following a power-law energy distribution, evolve suffering mainly synchrotron and relativistic Bremsstrahlung losses. In such a context, the spectrum of the particles is determined by the following transport equation (e.g. Khangulyan et al. 2007):

$\partial n(t, \gamma) / \partial t+\partial \dot{\gamma} n(t, \gamma) / \partial \gamma+n(t, \gamma) / \tau_{\mathrm{esc}}=Q(t, \gamma)$,

where $Q(t, \gamma)$ is the function for the particle injection, which takes place during the lifetime of the source $\left(\tau_{\text {life }}\right)$, estimated as $\gtrsim 100 \mathrm{yr} \approx 3 \times 10^{9} \mathrm{~s}$ (Garay et al. 2003). Here, $t$ is the time, and $\gamma$ is the lepton Lorentz factor. The age of the source is not well constrained, although it could hardly be significantly younger than $\sim 100 \mathrm{yr}$, the minimum jet-crossing time, since the shock has been probably active for most of this period while the shocked material was being displaced to its present location. Concerning $\tau_{\text {esc }}$, at this stage we take it as being the shortest diffusion timescale, i.e. that corresponding to the maximum energy particles. The time-derivative $\dot{\gamma}$ is a function accounting for all energy losses affecting leptons, i.e. basically synchrotron and relativistic Bremsstrahlung. The solution of Eq. (14) is:

$n(t, \gamma)=\frac{1}{\dot{\gamma}} \int_{\gamma}^{\gamma_{\mathrm{eff}}} Q\left(t-\tau, \gamma^{\prime}\right) \mathrm{e}^{-\tau\left(\gamma, \gamma^{\prime}\right) / \tau_{\mathrm{esc}}} \mathrm{d} \gamma^{\prime}$,

where

$t=\int_{\gamma}^{\gamma_{\mathrm{eff}}} \frac{\mathrm{d} \gamma^{\prime}}{\dot{\gamma}^{\prime}}$ and $\tau\left(\gamma, \gamma^{\prime}\right)=\int_{\gamma}^{\gamma^{\prime}} \frac{\mathrm{d} \gamma^{\prime \prime}}{\gamma^{\prime \prime}}$

The time dependence is shown in Eq. (15). For small $t, \gamma \lessgtr \gamma_{\mathrm{eff}}$; for larger $t, \gamma \ll \gamma_{\mathrm{eff}}$. Since $\gamma_{\mathrm{eff}}$ can be arbitrarily large, for $\gamma^{\prime}$ in Eq. (14) above the maximum Lorentz factor $Q$ will be zero. In addition, if $\tau$, being shorter than $\tau_{\text {cooling }}$, becomes larger than $\tau_{\text {esc }}$, the final particle spectrum will be affected by particle escape.

The computed particle energy distributions of primary and secondary leptons are shown in Fig. 3. As seen in the figure, for injection timescales $\tau_{\text {inj }} \sim 10^{9} \mathrm{~s}<\tau_{\text {life }}$, the particle distribution $n(t, \gamma)$ has already reached the steady regime, as expected from the fact that $\tau_{\text {cooling }} \sim 10^{9} \mathrm{~s}$. Moreover, the final particle energy distribution is not affected by $\tau_{\text {esc }}$ (which is actually a lower limit for the timescale of diffusion particle escape), since $\tau_{\text {esc }} \gtrsim \tau_{\text {cooling }}$ (see Fig. 2), i.e. particles will radiate inside the emitting region.

Regarding protons, their diffusion timescales and source lifetime are long enough to let them radiate almost all their energy in the hot spot. Moreover, the energy loss timescale is only slightly dependent on energy. This implies that proton energy distribution, in the context of our scenario, keeps almost the same spectral shape as that of the injected one, and reaches, as it is the case for leptons, the steady regime, without suffering significant impact from escape losses.

\subsection{Thermal radiation and ionization losses in the shocked region}

In the context of an outflow interacting with its environment, a strong shock of several hundreds $\mathrm{km} \mathrm{s}^{-1}$ can heat the shocked cloud material up to temperatures of $\sim 10^{7} \mathrm{~K}$, producing emission that would peak at soft X-rays. The heating of the medium would take place via shock compression, and ionization and Coulomb scattering of the thermal atoms/ions would occur due to the presence of relativistic leptons and protons. These soft $\mathrm{X}$-rays should be strongly absorbed by the surrounding medium, given the large hydrogen column density of the cloud. Hence, this radiation will be difficult to detect. In any case, this would lead to ionization of the cold material surrounding and/or embedded by the emitting region, which may affect to some extent the radio emission via free-free absorption. Since the observed radio spectrum seems to be non-thermal and we aim to obtain just first order estimates of the high-energy emission, we assume here that the overall effect of the free-free absorption is not dominant in the southern lobe of the source. Nevertheless, a more 

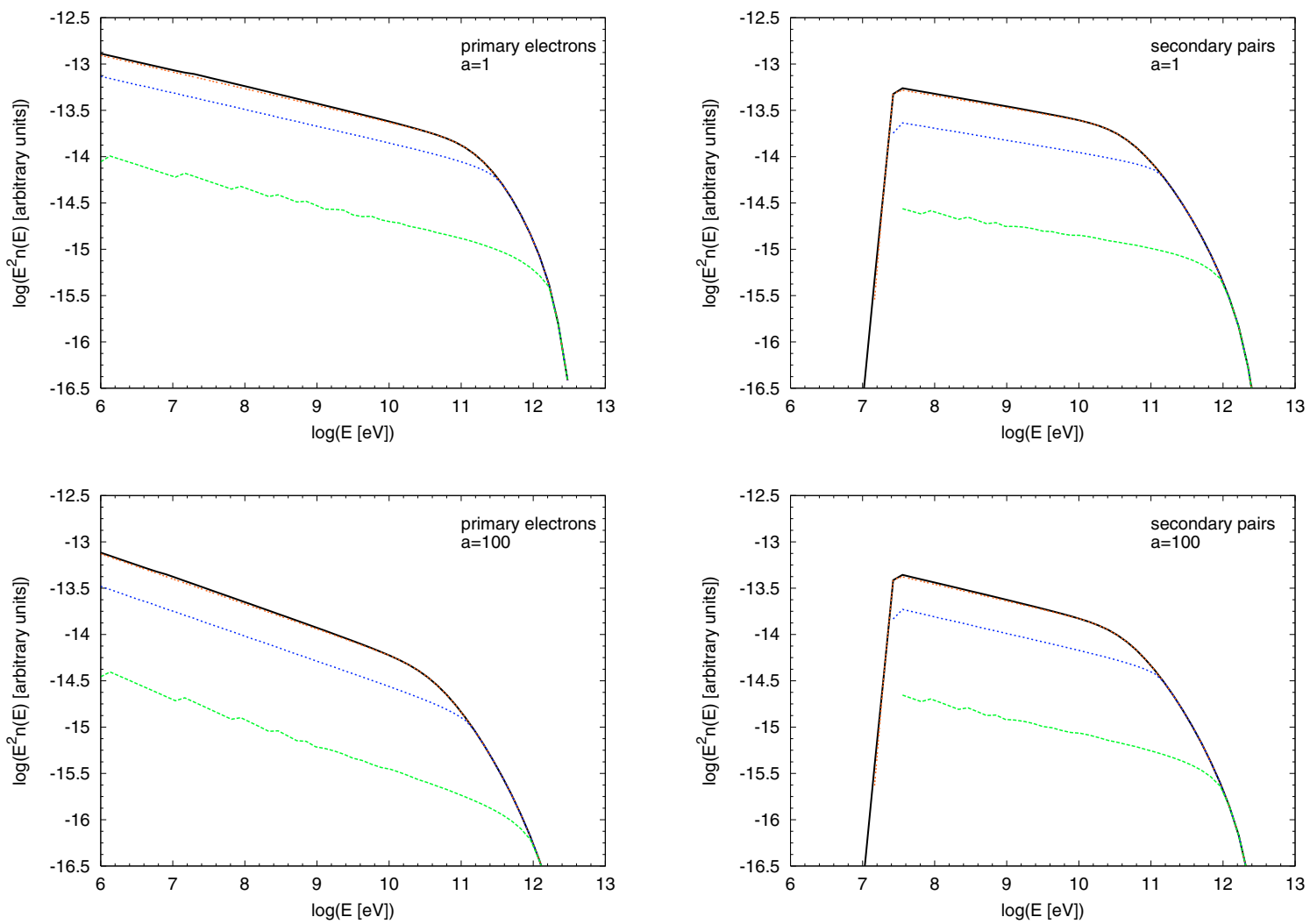

Fig. 3. SEDs of primary electrons and secondary pairs. Distributions for different particle injection timescales $\left(\tau_{\text {inj }}\right)$ are shown in each panel. Different curves correspond to the following values of $\tau_{\text {inj }}: 10^{7} \mathrm{~s}$ (long-dashed line, green), $10^{8} \mathrm{~s}$ (dot-dashed line, blue), $10^{9} \mathrm{~s}$ (dotted line, red) and $10^{10} \mathrm{~s}$ (solid line, black). Note that for ages $>10^{9} \mathrm{~s}$ the steady regime is reached (the curves appear in color in the online version of the paper only).

detailed characterization of the original radio spectrum would deserve further study. Another observational consequence of the ionization of the medium would be the detection of recombination lines, although their study is beyond the scope of this work.

It is worth mentioning that, if the emitting region were not significantly ionized by the jet termination shock, ionization losses would affect the particle energy distribution. The effect of this loss channel, with an energy loss rate that does not depend on energy, would be to harden the electron energy distribution at the energies where ionization losses are greater than those due to relativistic Bremsstrahlung, i.e. at Lorentz factors $\gamma<10^{3}$. The observed radio spectrum, however, is not particularly hard. This situation, nonetheless, might affect the northern lobe of the source.

\section{Production of gamma rays and lower energy radiation}

From the previous section it is clear that at the termination shock there will be particles energetic enough to produce gamma-ray emission. In this section we will use the particle distributions, ambient photon density and magnetic fields already estimated to calculate the non-thermal SEDs expected from the different particle interactions in the southern lobe of the radio source associated with IRAS 16547-4247.

\subsection{Leptonic interactions}

Synchrotron, relativistic Bremsstrahlung and IC emissivities for primary and secondary leptons were calculated using the standard formulae (e.g. Blumenthal \& Gould 1970; Pacholczyk 1970). Full Klein-Nishina cross section was used for the IC computation. The emission region was considered spherical, with a radius $r=r_{\text {lobe }}$. Figure 4 shows the results for the pure leptonic case $(a=0)$.

Focusing on the energy bands at which non-thermal emission may be detectable, we see that the high-energy emission is dominated by the relativistic Bremsstrahlung, with a peak of $\sim 10^{32} \mathrm{erg} \mathrm{s}^{-1}$ at $E_{\gamma} \sim 1 \mathrm{MeV}$. At energies $E_{\gamma} \gtrsim 1 \mathrm{GeV}$, the source presents luminosities of $\sim 10^{31} \mathrm{erg} \mathrm{s}^{-1}$, with a cutoff $\sim 10 \mathrm{GeV}$. At $\mathrm{X}$-rays, in the range $1-10 \mathrm{keV}$, the expected luminosities are about $10^{30} \mathrm{erg} \mathrm{s}^{-1}$, presenting a softening of the spectrum due to the high energy cutoff of the electron energy distribution.

\subsection{Hadronic interactions}

In the cases with $a=1$ and $a=100$, protons will have a significant contribution. The differential $\gamma$-ray emissivity generated through $\pi^{0}$-decay was calculated as

$q_{\gamma}\left(E_{\gamma}\right)=2 \int_{E_{\pi}^{\min }\left(E_{\gamma}\right)}^{\infty} \frac{q_{\pi^{0}}\left(E_{\pi}\right)}{\sqrt{E_{\pi}^{2}-m_{\pi}^{2} c^{4}}} \mathrm{~d} E_{\pi}$,

where $E_{\pi}^{\min }\left(E_{\gamma}\right)=E_{\gamma}+\frac{m_{\pi}^{2} c^{4}}{4 E_{\gamma}}$. Applying the $\delta$-function approximation for the differential cross section ${ }^{1}$

${ }^{1}$ This approximation considers only the most energetic neutral pion that is produced in the $p p$ reaction aside of a fireball composed by a certain number of less energetic $\pi$-mesons of each flavor. See the discussion in Pfrommer \& En $\beta$ lin (2004). 


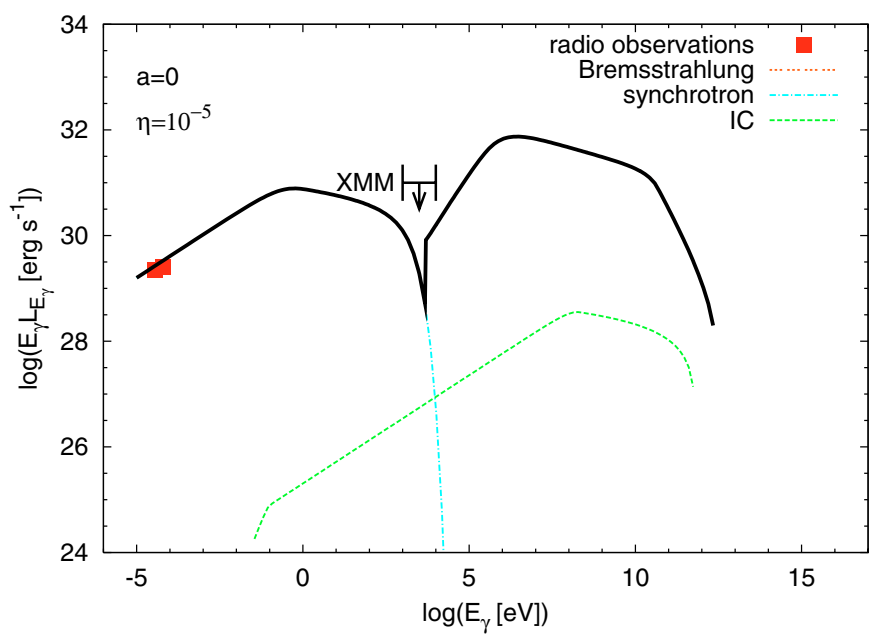

Fig. 4. SED for a pure leptonic case.

(Aharonian \& Atoyan 2000), the pion emissivity becomes

$$
\begin{aligned}
q_{\pi^{0}}\left(E_{\pi}\right)= & 4 \pi \int_{E_{\mathrm{th}}}^{\infty} \delta\left(E_{\pi}-\kappa E_{\mathrm{kin}}\right) J_{\mathrm{p}}\left(E_{\mathrm{p}}\right) \sigma_{\mathrm{pp}}\left(E_{\mathrm{p}}\right) \mathrm{d} E_{\mathrm{p}} \\
= & \frac{4 \pi}{\kappa} J_{\mathrm{p}}\left(m_{\mathrm{p}} c^{2}+\frac{E_{\pi}}{\kappa}\right) \sigma_{\mathrm{pp}}\left(m_{\mathrm{p}} c^{2}+\frac{E_{\pi}}{\kappa}\right)
\end{aligned}
$$

for proton energies greater than the energy threshold $E_{\mathrm{th}}=$ $1.22 \mathrm{GeV}$ and lower than $100 \mathrm{GeV}$. Here, $\kappa$ is the mean fraction of the kinetic energy $E_{\text {kin }}=E_{\mathrm{p}}-m_{\mathrm{p}} c^{2}$ of the proton transferred to a secondary meson per collision. For a broad energy region $(\mathrm{GeV}$ to $\mathrm{TeV}) \kappa \sim 0.17$. The total cross-section of the inelastic $p p$ collisions is given by Eq. (7). In Eq. (17) $J_{\mathrm{p}}\left(E_{\mathrm{p}}\right)=(4 \pi / c) n\left(E_{\mathrm{p}}\right)$ is the proton flux. For $E_{\mathrm{p}}>100 \mathrm{GeV}$, the equations given by Kelner et al. (2006) were used.

The specific luminosity can then be estimated as:

$E_{\gamma} L_{E_{\gamma}}=E_{\gamma}^{2} \int q_{\gamma}\left(E_{\gamma}\right) n(\boldsymbol{r}) \mathrm{d} \boldsymbol{r}$

where $n(\boldsymbol{r})$ is the ambient particle density.

Results from our calculations can be seen in Figs. 5 and 6. The hadronic emission is similar in luminosity to the relativistic Bremsstrahlung $\left(\sim 10^{32} \mathrm{erg} \mathrm{s}^{-1}\right)$, but extends up to higher energies $\sim 1 \mathrm{TeV}$. Primary electrons dominate the synchrotron and relativistic Bremsstrahlung emission in the case $a=1$, but for $a=100$ (see Fig. 4) the secondary leptons produce the most important contribution. In neither case does the IC play a significant role. Basically, the emission above $E_{\gamma} \sim 1 \mathrm{GeV}$ is due to neutral pion decays, whereas the X-ray and soft gamma rays are produced by relativistic Bremsstrahlung. For the cases $a=1$ and 100, the synchrotron and relativistic Bremsstrahlung components produced by secondary pairs are similar in spectral shape to those generated by primary electrons in the same cases, but with different behavior at low energies, due to the low-energy cutoff introduced by the minimum energy at which secondaries are injected.

\section{Discussion and summary}

Gamma-ray sources on the Galactic plane are usually associated with star-forming regions (Romero et al. 1999). However, the sources detected by EGRET are far more luminous than the sources that can be produced by massive YSOs according to our calculations. EGRET unidentified sources are most likely produced by pulsars, supernova remnants, massive stars

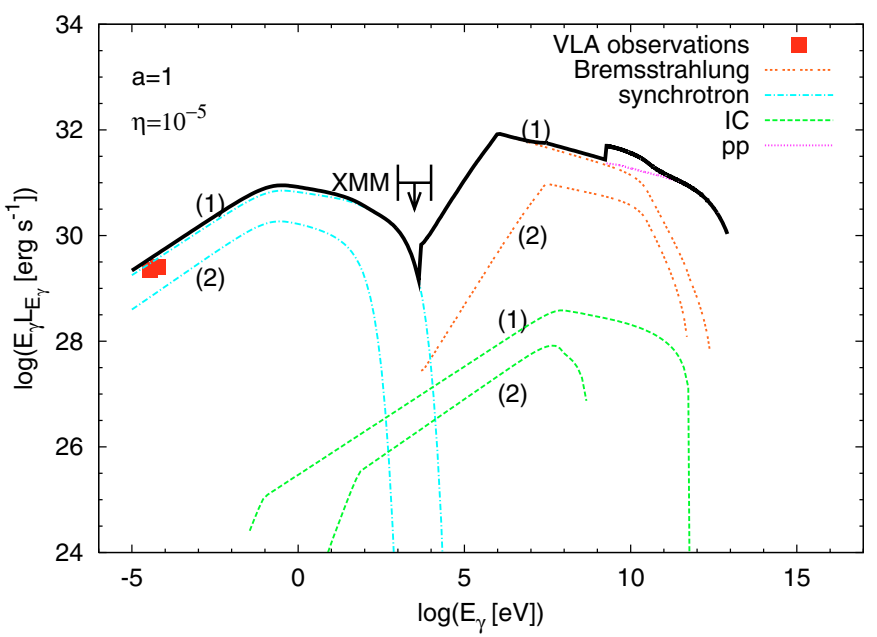

Fig. 5. SED for a mixed primary population of relativistic electrons and protons. We indicate with (1) and (2) the contribution from primary and secondary electrons, respectively.

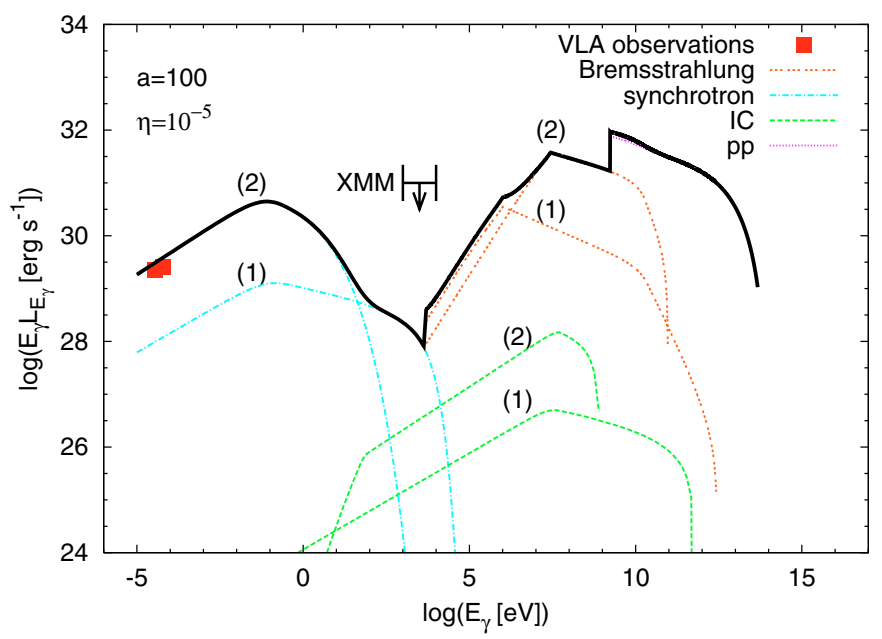

Fig. 6. Idem Fig. 5, but for a case dominated by relativistic protons.

(Romero 2001), and microquasars (Bosch-Ramon et al. 2005). The emission of massive YSOs falls below the sensitivity of EGRET, but they can be detected in principle by GLAST. At very high energies $\left(E_{\gamma} \gtrsim 100 \mathrm{GeV}\right)$, Cherenkov arrays such as HESS-II could detect a source like IRAS 16547-4247. Since such instruments might find the high-energy cutoff, valuable information could be obtained about the true efficiency of the acceleration process.

At X-rays, the source may be detectable by Chandra and XMM-Newton, probably as point-like, through a deep observation of the region. Briefly looking at archive XMM-Newton 30 ks observations of IRAS 16547-4247, there is not significant emission above the background from the southern lobe region. This yields an upper-limit for the $1-10 \mathrm{keV}$ luminosity of $\sim 10^{31} \mathrm{erg} \mathrm{s}^{-1}$ at the distance of $2.9 \mathrm{kpc}$. This value is not in contradiction with the luminosities of synchrotron and the relativistic Bremsstrahlung components expected in this energy range (see Figs. 4-6). Nevertheless, a deeper observation may detect radiation from this region. If the temperatures of the shocked material are high enough, a thermal component may be detectable in the X-rays as well. In the UV-band, the luminosity may still be higher than that produced by non-thermal particles, but could be difficult to detect. 
A relevant issue for the feasibility of the scenario discussed in this paper is that the non-thermal luminosities are well below the kinetic energy budget of the jet in IRAS 16547-4247. The jet mass-loss rate has been estimated by Garay et al. (2003) in $\sim 10^{-5} M_{\odot} \mathrm{yr}^{-1}$. With a velocity of $\sim 10^{3} \mathrm{~km} \mathrm{~s}^{-1}$, the jet kinetic luminosity is $\sim 3 \times 10^{36} \mathrm{erg} \mathrm{s}^{-1}$, more than three orders of magnitude larger than the non-thermal luminosity. Moreover, the energy density of the shocked thermal gas should be about two orders of magnitude larger than that of the non-thermal particles. Therefore, sustaining the non-thermal emission estimated in this work under reasonable assumptions does not seems to be a problem from the energetic point of view.

Summarizing, the fact that synchrotron radio emission has been detected from IRAS 16547-4247 indicates that there is conversion of energy of some sort to non-thermal particles. The high densities present in the region, as well as the relatively high kinetic luminosity of the jet, render it likely that radiation will be significantly generated by channels other than synchrotron radiation, such as relativistic Bremsstrahlung or even $p p$ interactions. After studying in a first-order approach a reasonable scenario for the non-thermal emitting region, we conclude that YSOs such as IRAS 16547-4247 could produce detectable non-thermal emission via synchrotron, relativistic Bremsstrahlung and $p p$ interactions from radio up to very high-energies. This opens a new window for studies of massive star formation in the near future.

Acknowledgements. We thank an anonymous referee for insightful comments and sugestions. A.T.A. and G.E.R. are supported by CONICET (PIP 5375) and the Argentine agency ANPCyT through Grant PICT 03-13291 BID 1728/OCAC. V.B-R. thanks the Max-Planck-Institut für Kernphysik for its support and kind hospitality. V.B-R., and J.M.P acknowledge support by DGI of MEC under grant AYA2004-07171-C02-01, as well as partial support by the European Regional Development Fund (ERDF/FEDER). V.B-R. gratefully acknowledges support from the Alexander von Humboldt Foundation.

\section{References}

Aharonian, F. A., \& Atoyan, A. M. 2000, A\&A, 362, 937

Bell, A. R. 1978, MNRAS, 182, 147

Blumenthal, G. R., \& Gould, R. J. 1970, Rev. Mod. Phys., 42, 237

Bonnell, I. A., Bate, M. R., \& Zinnecker, H. 1998, MNRAS, 298, 93

Bosch-Ramon, V., Romero, G. E., \& Paredes, J. M. 2005, A\&A, 429, 267

Brooks, K., Garay G., Mardones, D., \& Bronfman, L. 2003, ApJ, 594, L131

Garay, G., Brooks, K., Mardones, D., \& Norris, R. P. 2003, ApJ, 537, 739

Ginzburg, V. L., \& Syrovatskii, S. I. 1964, The Origin of Cosmic Rays (New York: Pergamon Press)

Khangulyan, D., Hnatic, S., Aharonian F., \& Bogovalov S. 2007, MNRAS, in press

Kelner, S. R., Aharonian, F. A., \& Vugayov, V. V. 2006, Phys. Rev. D, 74, 034018 Mannheim, K., \& Schlickeiser, R. 1994, A\&A, 286, 983

Martí, J., Rodríguez, L. F., \& Reipurth, B. 1995, ApJ, 449, 184

Pacholczyk, A. G. 1970, Radio Astrophysics (San Francisco: Freeman)

Pfrommer, C., \& En $\beta$ lin, T. A. 2004, A\&A, 413, 17

Protheroe, R. J. 1999, in Topics in Cosmic-Ray Astrophysics, 1999, 247 [arXiv: astro-ph/9812055]

Rodriguez L. F., Garay G., Brooks, K., \& Mardones, D. 2005, ApJ 626, 953

Romero, G. E. 2001, in The Nature of Unindentified Galactic High-Energy Gamma-Ray Sources, ed. A. Carraminana, O. Reimer, \& D. Thompson (Dordrecht: Kluwer Academic Publishers), 65

Romero, G. E., Benaglia, P., \& Torres, D. F. 1999, A\&A, 348, 868

Shu, F. H., Adams, F. C., \& Lizano, S. 1987, ARA\&A, 25, 23 